\title{
Metagenomic survey of methanesulfonic acid (MSA) catabolic genes in an Atlantic Ocean surface water sample and in a partial enrichment
}

\author{
Ana C Henriques ${ }^{1}$, Rui MS Azevedo ${ }^{1}$, Paolo De Marco ${ }^{\text {Corresp. } 1}$ \\ 1 Instituto de Investigação e Formação Avançada em Ciências e Tecnologias da Saúde (IINFACTS), CESPU, Gandra PRD, Portugal \\ Corresponding Author: Paolo De Marco \\ Email address: paolo.marco@iscsn.cespu.pt
}

Methanesulfonic acid (MSA) is a relevant intermediate of the biogeochemical cycle of sulfur and environmental microorganisms assume an important role in the mineralization of this compound. Several methylotrophic bacterial strains able to grow on MSA have been isolated from soil or marine water and two conserved operons, msmABCD coding for MSA monooxygenase and msmEFGH coding for a transport system, have been repeatedly encountered in most of these strains. Homologous sequences have also been amplified directly from the environment or observed in marine metagenomic data, but these showed a base composition ( $\mathrm{G}+\mathrm{C}$ content) very different from their counterparts from cultivated bacteria. The aim of this study was to understand which microorganisms within the coastal surface oceanic microflora responded to MSA as a nutrient and how the community evolved in the early phases of an enrichment by means of metagenome and gene-targeted amplicon sequencing. From the phylogenetic point of view, the community shifted significantly with the disappearance of all signals related to the Archaea, the Pelagibacteraceae and phylum SAR406, and the increase in methylotroph-harboring taxa, accompanied by other groups so far not known to comprise methylotrophs such as the Hyphomonadaceae. At the functional level, the abundance of several genes related to sulfur metabolism and methylotrophy increased during the enrichment and the allelic distribution of gene $m s m A$ diagnostic for MSA monooxygenase altered considerably. Even more dramatic was the disappearance of MSA import-related gene $m s m E$, which suggests that alternative transporters must be present in the enriched community and illustrate the inadequacy of $m s m E$ as an ecofunctional marker for MSA degradation at sea. 
1 Metagenomic survey of methanesulfonic acid (MSA) catabolic genes in an

2 Atlantic Ocean surface water sample and in a partial enrichment

3 Ana C. Henriques, Rui M. S. Azevedo and Paolo De Marco*

4 CESPU, Instituto de Investigação e Formação Avançada em Ciências e Tecnologias da Saúde

5 (IINFACTS), Gandra PRD, Portugal

6

7 * email address: paolo.marco@iscsn.cespu.pt 


\section{Abstract}

9 Methanesulfonic acid (MSA) is a relevant intermediate of the biogeochemical cycle of sulfur and

environmental microorganisms assume an important role in the mineralization of this compound.

Several methylotrophic bacterial strains able to grow on MSA have been isolated from soil or marine water and two conserved operons, $m s m A B C D$ coding for MSA monooxygenase and msmEFGH coding for a transport system, have been repeatedly encountered in most of these strains. Homologous sequences have also been amplified directly from the environment or observed in marine metagenomic data, but these showed a base composition $(\mathrm{G}+\mathrm{C}$ content $)$ very different from their counterparts from cultivated bacteria. The aim of this study was to understand which microorganisms within the coastal surface oceanic microbiota responded to MSA as a nutrient and how the community evolved in the early phases of an enrichment by means of metagenome and gene-targeted amplicon sequencing. From the phylogenetic point of view, the community shifted significantly with the disappearance of all signals related to the Archaea and phylum SAR406 and almost all the Pelagibacteraceae, and the increase in methylotroph-harboring taxa, accompanied by other groups so far not known to comprise methylotrophs such as the Hyphomonadaceae. At the functional level, the abundance of several genes related to sulfur metabolism and methylotrophy increased during the enrichment and the allelic distribution of gene $m s m A$ diagnostic for MSA monooxygenase altered considerably. Even more dramatic was the disappearance of MSA import-related gene $m s m E$, which suggests that alternative transporters must be present in the enriched community and illustrate the inadequacy of $m s m E$ as an ecofunctional marker for MSA degradation at sea. 
Abbreviations: MSA $=$ methanesulfonic acid; MSAMO $=$ MSA monooxygenase; DMS $=$ dimethylsulfide; SSM = Sargasso Sea metagenome; GOS = Global Ocean Sampling.

\section{Introduction}

It is known that methanesulfonic acid (MSA) has been produced during millennia in the atmosphere by the oxidation of dimethylsulfide (DMS) that escapes from the seawater surface (Andreae, 1986; Hynes, Wine \& Semmes, 1986; Mihalopoulos et al., 1992; Koga \& Tanaka, 1993; Kelly \& Murrell, 1999). DMS is mainly a byproduct of the degradation of marine photosynthetic organisms (Todd et al., 2011) and is the major component of marine emissions of volatile sulfur (Gondwe et al., 2003). Due to the huge scale of this biogeochemical process, very significant amounts (est. $10^{10} \mathrm{~kg}$ ) of MSA form annually and deposit back onto the sea or land surfaces (Charlson et al., 1987; Kelly \& Murrell, 1999; Gondwe et al., 2003). MSA has not been found accumulating in any environment (apart from perennial ices (Legrand \& Feniet-Saigne, 1991; Whung et al., 1994)), which means that this compound is readily degraded in Nature. Several methylotrophic bacterial strains have been isolated that can use MSA as sole source of carbon and energy (Kelly \& Baker, 1990; Thompson, Owens \& Murrell, 1995; De Marco et al., 2000, 2004; Baxter et al., 2002; Moosvi et al., 2005) while other microbes are known to use this molecule just as a sulfur supply (Kelly \& Murrell, 1999). An operon (msmABCD) encoding a heteromeric monooxygenase (MSA monooxygenase, or MSAMO) and another operon (msmEFGH) encoding uptake proteins have been found in several MSA-utilizing strains (De Marco et al., 1999; Baxter et al., 2002; Jamshad et al., 2006; Henriques \& De Marco, 2015a,b) and in a marine bacterium isolated from Western Pacific surface waters (Oh et al., 2010). 
51 All Proteobacterial strains known to use MSA methylotrophically that have been analyzed at the

52 molecular level have shown to possess gene $m s m A$ and most of them carry gene $m s m E$ (Henriques

53 \& De Marco, 2015a,b). By contrast the only Actinobacterial strain known to grow on MSA as a

54 carbon source (Rhodococcus str. RD6.2 (De Marco et al., 2004)) harbors an alternative gene, ssuD,

55 coding for an enzyme previously associated solely to non-methylotrophic MSA utilization

56 (Eichhorn, Van Der Ploeg \& Leisinger, 1999; Endoh et al., 2003), plus some broad-range

57 alkanesulfonate monooxygenases (Henriques \& De Marco, 2015c). Homologs of the msm genes

58 and operons have also been directly amplified from environmental DNA (Baxter et al., 2002;

59 Henriques \& De Marco, 2015a), retrieved by metagenomic sequencing projects (Leitão, Moradas-

60 Ferreira \& De Marco, 2009) or found highly expressed in metatranscriptomic analyses of surface

61 seawater (Gifford et al., 2013). Dozens of hits for $m s m A$ gene can also be recovered from the

62 recently published Tara Oceans project data (Sunagawa et al., 2015) and one apparent msm double

63 operon was found in one of the single-cell genomes (Alphaproteobacterium SCGC AAA536-B06)

64 sequenced from the Mediterranean Sea (Swan et al., 2013). Among the MSAMO enzyme

65 components, the ferrodoxin (MsmC) and the FAD-binding NADH-dependent reductase (MsmD)

66 are very similar to analogous components of unrelated oxygenases while MsmB, like many other

67 examples of hydroxylase beta subunits, shows poor sequence conservation (De Marco et al., 1999;

68 Baxter et al., 2002). On the contrary, alpha subunits of MSAMO hydroxylases (MsmA) show

69 strong conservation and a peculiar 26-amino acid-long spacer within the Rieske-type [2Fe-2S]-

70 binding motif. Among the polypeptides involved in the import of MSA into the cell, MsmE is the

71 one that, in the proposed model, binds MSA in the periplasm. For these reasons, genes $m s m A$ and

$72 m s m E$ have been selected as promising markers for MSA-utilizing bacteria in the environment.

73 However, most likely owing to lower sequence conservation, designing robust primer pairs for 
74 gene E and obtaining bona fide $m s m E$ amplicons has been much less successful than with gene $A$ 75 (Henriques \& De Marco, 2015a). More in general, most of these genes obtained directly from 76 marine water samples were low or very low in $\mathrm{G}+\mathrm{C}$ content $(36-48 \%)$ while cultivated strains 77 obtained from both soil, estuary and seawater had $\mathrm{G}+\mathrm{C}$ content levels in the $47-66 \%$ range. This 78 dichotomy is not unique to MSA-utilizers or msm genes: the single-cell genomes retrieved by 79 Swan et al. (Swan et al., 2013) showed clear signs of streamlining and much lower GC\% than 80 cultivated marine strains, which may be a reflection of the generalized oligotrophic traits of 81 unculturable bacteria versus the copiotrophic nature of cultivated types. Consistent with all these observations is the idea that laboratory isolates are no proper representatives of natural populations and that the only way to obtain a truthful picture of microbial communities is through in situ physiological observation and/or direct molecular investigation.

85 In this work we analyzed phylogenetically and functionally a surface coastal seawater sample from the Atlantic Ocean and repeated the analysis after partial enrichment with MSA as sole added

87 source of carbon, energy and sulfur. Amplicon survey analyses on functional markers for MSA degradation, genes $m s m A$ and $m s m E$, were also carried out. 
90

91

92

93

94

95

96

97

98

\section{Material \& methods}

\section{Seawater sample collection and metagenomic DNA isolation}

Atlantic Ocean surface water was collected on Dec $3^{\text {rd }}, 2014$ along the coast of Leça da Palmeira, Portugal (approximate coordinates 41.226956, -8.720528). Approximately 15 liters of seawater were collected off the rocky shore at rising tide into clean bottles, which were immediately transported to the lab in an isothermal bag with ice packs. Before starting the procedures, the bottles were shaken in order to homogenize the samples. Three fractions of $0.5 \mathrm{~L}$ were filtered through $1.2 \mu \mathrm{m}$ glass fiber filters, which were immediately wrapped in aluminum foil and stored at $-80^{\circ} \mathrm{C}$ for later quantification of chlorophyll. The determination of chlorophyll $a$ concentration was performed through a spectrophotometric method as previously described (Inag, 2009). Chlorophyll $a$ concentration was calculated through the Jeffrey and Humphrey equation (Jeffrey \& Humphrey, 1975). Measures for $\mathrm{pH}$ and conductivity were also performed. Two fractions of 5 $\mathrm{L}$ of the seawater sample were filtered in parallel through $1.2 \mu \mathrm{m}, 0.45 \mu \mathrm{m}$ and $0.2 \mu \mathrm{m}$ filters in succession. The filters from one $5 \mathrm{~L}$ fraction were immediately used for DNA extraction using the PowerWater DNA Isolation Kit (MO BIO Laboratories, Inc.) according to the manufacturer's instructions.

\section{Marine enrichment with MSA and metagenomic DNA isolation}

The biomass retained on the filters from the second $5 \mathrm{~L}$ fraction was resuspended in the last 200 $\mathrm{mL}$ of the ocean water sample. The filters were removed and $1 \mathrm{~mL}$ of alkaline $(\mathrm{pH} 8)$ sodium methanesulfonate (MSA) $1 \mathrm{M}$ was added to the suspension (5 mM final concentration). Similar 
112 amounts of alkaline MSA were used to spike the enrichment at days 7, 9 and 12. The suspension

113 was incubated aerobically at room temperature (ca. $20^{\circ} \mathrm{C}$ ) in the dark, continuously mixed by a

114 magnetic stirrer. This schedule was deliberately maintained during 16 days, with no

115 subculturing, in order to produce just a partially enriched culture. The biomass at the end of the

116 enrichment process was collected by centrifugation and DNA was extracted with PowerWater

117 DNA Isolation Kit (MO BIO Laboratories, Inc., with adaptations to accommodate biomass in a

118 pellet rather than on a filter). For convenience, the seawater sample (time 0) and the

119 corresponding enrichment (E) culture will be designated SCD0 and SCDE, respectively. This

120 study was limited to the observation of the evolution of the microbial community of a single

121 sample and as such the following results are to be considered exploratory.

\section{Whole metagenome sequencing}

124 The metagenomes (from SCD0 and SCDE samples) were sequenced at Molecular Research LP

125 (Shallowater, TX, USA). Paired-end sequencing libraries were prepared (2x101 bp) and

126 sequencing was performed using the Illumina HiSeq2500 platform. The libraries were prepared

127 using Nextera DNA Sample preparation kit (Illumina) following the manufacturer's user guide.

128 The initial concentration of DNA was evaluated using the Qubit ${ }^{2}$ dsDNA HS Assay Kit (Life

129 Technologies). The samples were then diluted accordingly to achieve the recommended DNA

130 input of $50 \mathrm{ng}$ at a concentration of $2.5 \mathrm{ng} / \mu \mathrm{L}$. Subsequently, the samples underwent

131 fragmentation, addition of adapter sequences and PCR amplification ( 5 cycles) during which a

132 unique index was added to each sample. The average library size was determined using the

133 Agilent 2100 Bioanalyzer (Agilent Technologies). The libraries were then pooled in equimolar 
134 ratios at $2 \mathrm{nM}$ and $5 \mu \mathrm{L}$ of the library pool was clustered using the cBot (Illumina) and

135 sequenced paired-end for 200 cycles using the HiSeq 2500 system (Illumina).

136 The quality of the sequencing reads from both libraries was assessed using FastQC on Galaxy

137 web-based platform (https://usegalaxy.org/). The libraries were also checked for human

138 contamination with Kraken Metagenomics (Zaharia et al., 2011; Wood \& Salzberg, 2014) at

139 Illumina BaseSpace (http://basespace.illumina.com/home/index). As the resulting values for the

140 presence of human sequences were very low $(0.15 \%$ for sample SCD0 and $0.04 \%$ for sample

141 SCDE), no filtering was performed before the subsequent steps of analysis (general statistics are 142 described in Table S1).

143 Sequencing data for the two samples, SCD0 and SCDE, were submitted to the European

144 Nucleotide Archive (http://www.ebi.ac.uk/ena) under project number PRJEB9018 and sample

145 accession numbers ERS700852 and ERS700853, respectively. The analyses of the metadata

146 were performed through the EBI Metagenomics service pipeline

147 (https://www.ebi.ac.uk/metagenomics/pipelines/2.0 (Mitchell et al., 2015a)) that includes a

148 quality control step, a taxonomic analysis step based on 16S rDNA sequences and a functional

149 analysis of predicted protein coding sequences using the InterPro resource (Mitchell et al., 150 2015b).

151 Biom files containing phylogenetic classification information provided by EBI metagenomics

152 were used to construct rarefaction curves using MEGAN (version 5.10.6; (Huson, Mitra \&

153 Ruscheweyh, 2011)). Phylogenetic data were also used to estimate the alpha diversity of the two

154 samples (Shannon index (Shannon, 1948), evenness (Mulder et al., 2004) and Chao species

155 estimator (Chao, 1984)). For beta diversity analysis, Jaccard (Jaccard, 1912), Kulczynski (Faith, 
156 Minchin \& Belbin, 1987), and Chao (Chao, Chazdon \& Shen, 2005) indices were calculated

157 through the Vegan package (Oksanen et al., 2015). Bray-Curtis dissimilarity index (Bray \&

158 Curtis, 1957) was calculated starting from relative abundances. Only significant differences in

159 phylogenetic or functional composition were retained (Fisher's exact / $\chi^{2}$ test with a significance

160 level of 0.05 with a Bonferroni correction on the number of comparisons, in order to minimize

161 false positives; code was adapted from Metastats (White, Nagarajan \& Pop, 2009)).

162 Assembled metagenomic data from both samples were also submitted to the DOE Joint Genome

163 Institute's Integrated Microbial Genome Metagenomic Expert Review (IMG/MER) annotation

164 pipeline (http://img.jgi.doe.gov/) for functional and taxonomic annotation (Markowitz et al.,

165 2014) (SCD0: GOLD Analysis Project Id Ga0069134 / biosample ID Gb0111627; SCDE:

166 GOLD Analysis Project Id Ga0069135 / biosample ID Gb0111630). Before submission, reads

167 were quality checked (FastQC for quality control at Galaxy for trimming and filtering): only

168 sequences with quality scores equal or higher than 20 over $95 \%$ or more of the nucleotides were

169 maintained. For each sample, forward and reverse sequence files were merged and assembled

170 using Megahit (Li et al., 2015) (general statistics are reported in Table S2). A further analysis

171 was performed at MG-RAST (Meyer et al., 2008) using the subsystems approach (Overbeek et

172 al., 2005): metagenome SCD0 was submitted under sample $\mathrm{n}^{\circ} 4698364.3$ and SCDE under

173 sample $\mathrm{n}^{\mathrm{o}}$ 4698363.3. Binning was performed using MetaBat v0.25.4 (Kang et al., 2015) and the

174 bins obtained were analyzed by MG-RAST.

175 A flowchart with the major steps of the analysis is available in Fig. S1. 
$m s m A$ and $m s m E$ amplicon surveys

178 In order to obtain DNA amounts sufficient for the subsequent analyses, the REPLI-g® MiniKit

179 (QIAGEN) was used to amplify the DNA from each metagenomic sample, according to the

180 manufacturer's instructions. Negative controls starting from ultrapure nuclease-free water

181 yielded no product.

182 The amplified metagenomes were tested with primers directed to genes $m s m A$ and $m s m E$. As

183 amplicons with less than $400 \mathrm{bp}$ were needed for Ion Torrent sequencing, primer pairs

184 SarA139fwd/SarA488rev and SarE322fwd/SarE704rev (Table S3) were employed in order to

185 obtain amplicons with 349 bp and 382 bp, respectively. However, when amplification was

186 performed directly with these primers, the signals obtained were too weak or absent. As such, a

187 nested PCR approach was carried out. The first round was performed using primer sets

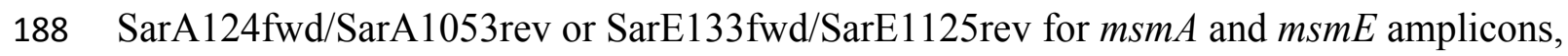

189 respectively (Table S3). Some parameters had to be adjusted in order optimize the reactions, and

190 the successful conditions are reported in Table S4. Negative controls received PCR water instead

191 of DNA. Positive controls contained DNA from Sargasso Sea Metagenome clone EF103447

192 (Leitão, Moradas-Ferreira \& De Marco, 2009). Only primers directed to the low-GC Sargasso

193 Sea Metagenome $m s m$ sequences had previously been successful at amplifying these genes from

194 a seawater metagenomic sample from the same location (Henriques \& De Marco, 2015a).

195 Accordingly, the primers used in this work were based on the known Sargasso Sea Metagenome

196 msm sequences. Amplification products were submitted for sequencing at Stabvida Lda.

197 (Caparica, Portugal). After determining the exact PCR product concentrations with Qubit 2.0

198 Fluorometer (Invitrogen) and Qubit dsDNA BR Kit (Invitrogen), platform-specific barcoded

199 adapters were added to each sample in the preparation of libraries with KAPA Library 
200 Preparation Kit (Kapa Biosystems) and NEXTflex ${ }^{\text {TM }}$ DNA Barcodes for Ion PGM (Bioo

201 Scientific). Samples were sequenced on an Ion Torrent Personal Genome Machine (PGM, Life

202 Technologies, Thermo Fisher Scientific) using Ion PGM ${ }^{\mathrm{TM}}$ Hi-Q Sequencing Kit reagents. Reads

203 were provided free from barcodes and adapters sequences.

204 Sequencing data from $m s m A$ amplicons, SCD0-A and SCDE-A sets, and $m s m E$ amplicons,

205 SCD0-E set, were submitted to the European Nucleotide Archive (http://www.ebi.ac.uk/ena)

206 under sample accession numbers ERS954926, ERS954925 and ERS954927, respectively, within

207 the metagenomic study PRJEB9018.

208 The overall quality scores of the output data from the Ion Torrent sequencing was assessed using

209 FastQC on Galaxy web-based platform. Sequencing reads were then trimmed and filtered based

210 on length and quality. For the $m s m A$ amplicon, reads ranging from 300 to 365 bp were selected;

211 for the $m s m E$ amplicon, sequences with length between 335 to $420 \mathrm{bp}$ were retained. In the

212 quality filtering step, sequences with quality lower than 20 over more than $15 \%$ of the

213 nucleotides were discarded. Chimeric sequences were removed with Chimera Check (Edgar et

214 al., 2011) available in the FunGenePipeline (Fish et al., 2013). The resulting files were submitted

215 to FrameBot (Wang et al., 2013) for translation and frameshift correction (FunGenePipeline).

216 Sequences containing stop and/or undefined codons were discarded (general statistics are

217 reported in Table S5). For each dataset, protein sequences were aligned with hmmalign

218 (HMMER3 (Eddy, 2011)) and clustered by complete linkage clustering (mcClust (Loewenstein

219 et al., 2008), FunGenePipeline). Rarefaction (FunGenePipeline (Fish et al., 2013)) was based on

220 data from the clust file at 0.03 distance. Conservation analysis of the MsmA and MsmE

221 predicted protein sequences was performed on alignments generated by hmmalign (HMMER3)

222 and analyzed by Jalview (Waterhouse et al., 2009). Alpha diversity analysis (Shannon index, 
223 evenness and Chao species estimator) was performed on clustering values obtained at 0.03

224 distance for each set of results. Beta diversity (Bray-Curtis, Jaccard, Kulczynski, and Chao

225 indices) between SCD0-A and SCDE-A sequence sets was computed on the clustered (0.03

226 distance) aligned MsmA predicted sequences. For Bray-Curtis, relative cluster abundances were

227 employed. The representative sequence for each cluster was obtained using Representative

228 Sequences (FunGenePipeline). In this case, distance cutoff values were chosen in order to obtain

229 in the region of 20-30 clusters from each dataset (0.15 for SCD0-A / SCDE-A and 0.10 for

$230 \mathrm{SCD}$-E). The nucleotide sequences corresponding to these cluster-representative MsmA

231 sequences were retrieved and used to infer phylogenetic trees by Maximum Likelihood with 100

232 bootstrap iterations (as implemented in PhyML (Guindon et al., 2010) at Phylogeny.fr (Dereeper

233 et al., 2008)). A flowchart with the major steps of this analysis is available in Fig. S2. 


\section{Results}

\section{Characterization of the samples}

236 The seawater sample collected for this study was at a temperature of $15.5^{\circ} \mathrm{C}$ and $3.34 \%$ salinity

237 (conductivity $47.9 \mathrm{mS} / \mathrm{cm}$ ). The $\mathrm{pH}$ value was 8.07 and the concentration of chlorophyll $a$ was

$2381.06 \mathrm{mg} / \mathrm{m}^{3}$.

239 Optical density (at $600 \mathrm{~nm}$ ) of the filtered sample was 0.606 at time 0 (SCD0), dropped to 0.415

240 at day 2 and rose towards the last days of incubation to 0.758 (SCDE). During the enrichment

241 process the suspension progressively lost its original greenish hue.

\section{Analysis of phylogenetic composition}

245 Despite the fact that sample SCDE underwent just a partial enrichment with no dilution or

246 subculturing, this was enough for the $\mathrm{G}+\mathrm{C}$ content of the community's metagenome to shift from

$24743.30 \%$ (SCD0) to 53.29\% (SCDE). Phylogenetic composition analysis was performed by EBI

248 Metagenomics on the metagenomic data, based on prokaryotic rRNA gene sequences.

249 Rarefaction curves were generated from these results for both metagenomes (Fig. S3). These

250 curves show that a reasonable coverage of the communities' composition was achieved:

251 however, neither reaches a definite plateau meaning that deeper sequencing levels would have

252 been desirable.

253

254 Phylogenetically, the community before the enrichment was composed primarily by taxa typical 255 of surface seawater, namely Alphaproteobacteria of the Pelagibacteraceae and the 
256 Rhodobacteraceae, Gammaproteobacteria of the Halomonadaceae and Piscirickettsiaceae,

257 Archaea of the Crenarchaeota (with prominence for Nitrosopumilus) and the Euryarchaeota

258 (Thermoplasmata Marine group II), Bacteroidetes of the Flavobacteriaceae and the uncultured

259 marine phylum SAR406 (Fig.s 1 and 2).

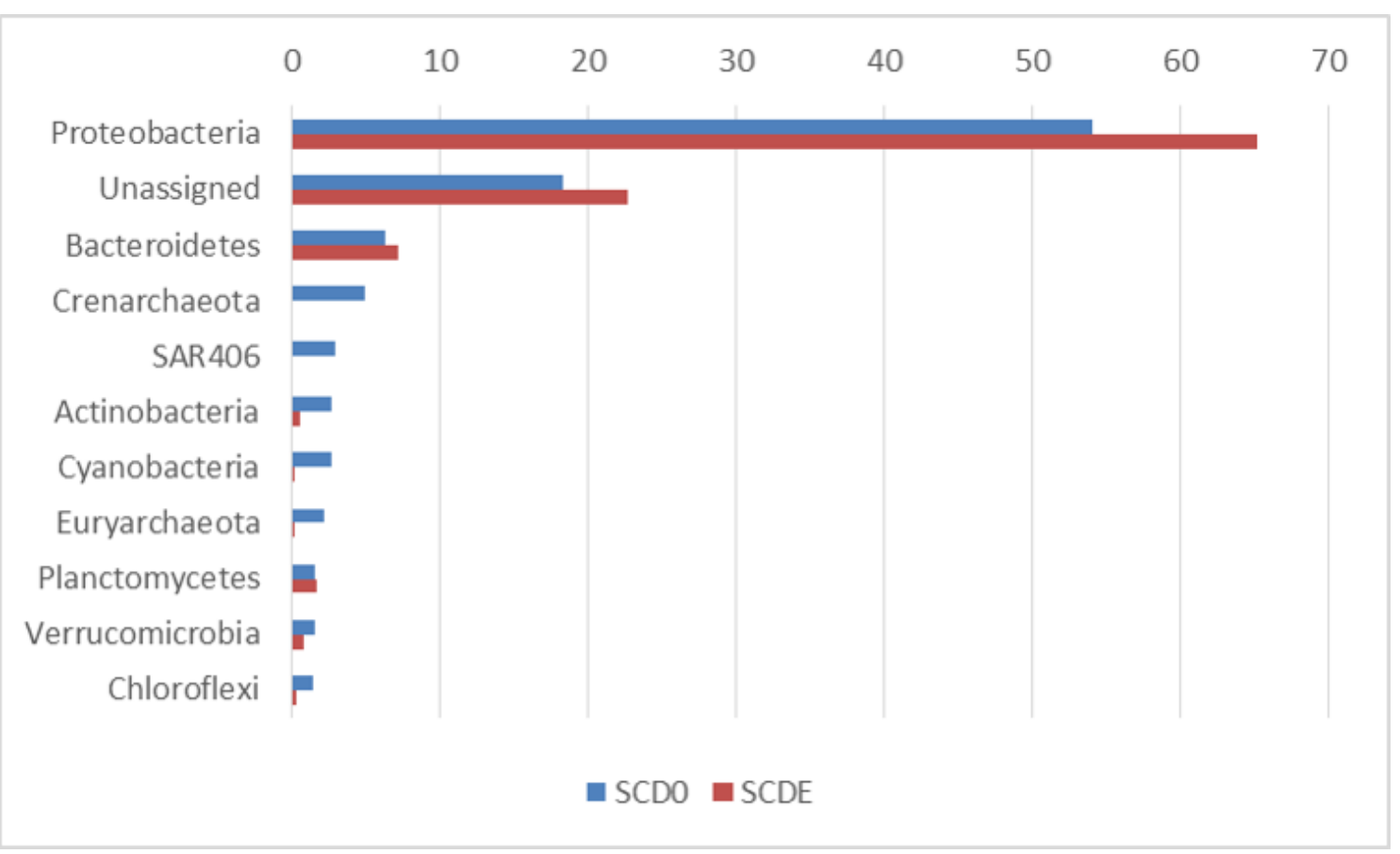

261 Figure 1. Phylogenetic composition (phyla) of the two metagenomes, SCD0 and SCDE (in

262 percentage). Only phyla with abundance $\geq 1 \%$ in either sample are shown (based on EBI

263 Metagenomics analysis of the data).

264 Clearly, some of these groups declined or disappeared during our enrichment program (Fig.s 1, 2

265 and 3): all the Archaea and SAR406, the Pelagibacteraceae, Halomonadaceae,

266 Flavobacteriaceae (a family known to harbor methylotrophic species (De Marco et al., 2004;

267 Azra Moosvi et al., 2005; Boden et al., 2008; Madhaiyan et al., 2010) and Variovorax paradoxus

268 (Comamonadaceae) a known methylotroph (Anesti et al., 2005). Other groups thrived: the

269 Rhodobacteraceae and the Hyphomonadaceae within the Alphaproteobacteria, the

270 Oceanospirillaceae, the Piscirickettsiaceae, 2 families of Alteromonadales and genus 
271 Alcanivorax within the Gammaproteobacteria, the Saprospiraceae within the Bacteroidetes. It is

272 worth noticing that: fam. Rhodobacteraceae includes several methylotrophic species and namely

273 marine MSA-degrader Marinosulfonomonas (Thompson, Owens \& Murrell, 1995); fam.

274 Piscirickettsiaceae includes the genus Methylophaga, a known methylotrophic taxon relevant in 275 surface sea waters (Neufeld et al., 2008). Indeed, the signal for Methylophaga itself at the genus

276 level shot up from below detection to 82 hits; genus Alcanivorax, which contains marine

277 representatives that have previously been found in a dimethylsulfide-based enrichment (Schäfer, 278 2007) and one known potentially methylotrophic species (A. borkumensis strain SK2 -

279 http://www.ebi.ac.uk/biomodels-main/BMID000000083743). Despite the fact that to our

280 knowledge no methylotrophic species is known among the Hyphomonadaceae, the considerable

281 increase in its abundance after the enrichment (225x) seem to suggest a possible involvement of 282 members of this group in the turnover of MSA. Other rarer taxa that showed sizeable abundance 283 increases after the enrichment were the Cryomorphaceae (Bacteroidetes), among the 284 Gammaproteobacteria several branches within the Alteromonadales, several groups within the 285 Oceanospirillales like the Oceanospirillaceae (and species Neptuniibacter caesariensis), the 286 Oleiphilaceae, genus Halomonas, and rather surprisingly the Enterobacteriaceae. Notably, 287 known methylotrophic-containing taxa like the Hyphomicrobiaceae (Alphaproteobacteria) and 288 Methylotenera mobilis (Betaproteobacteria) also increased significantly. 


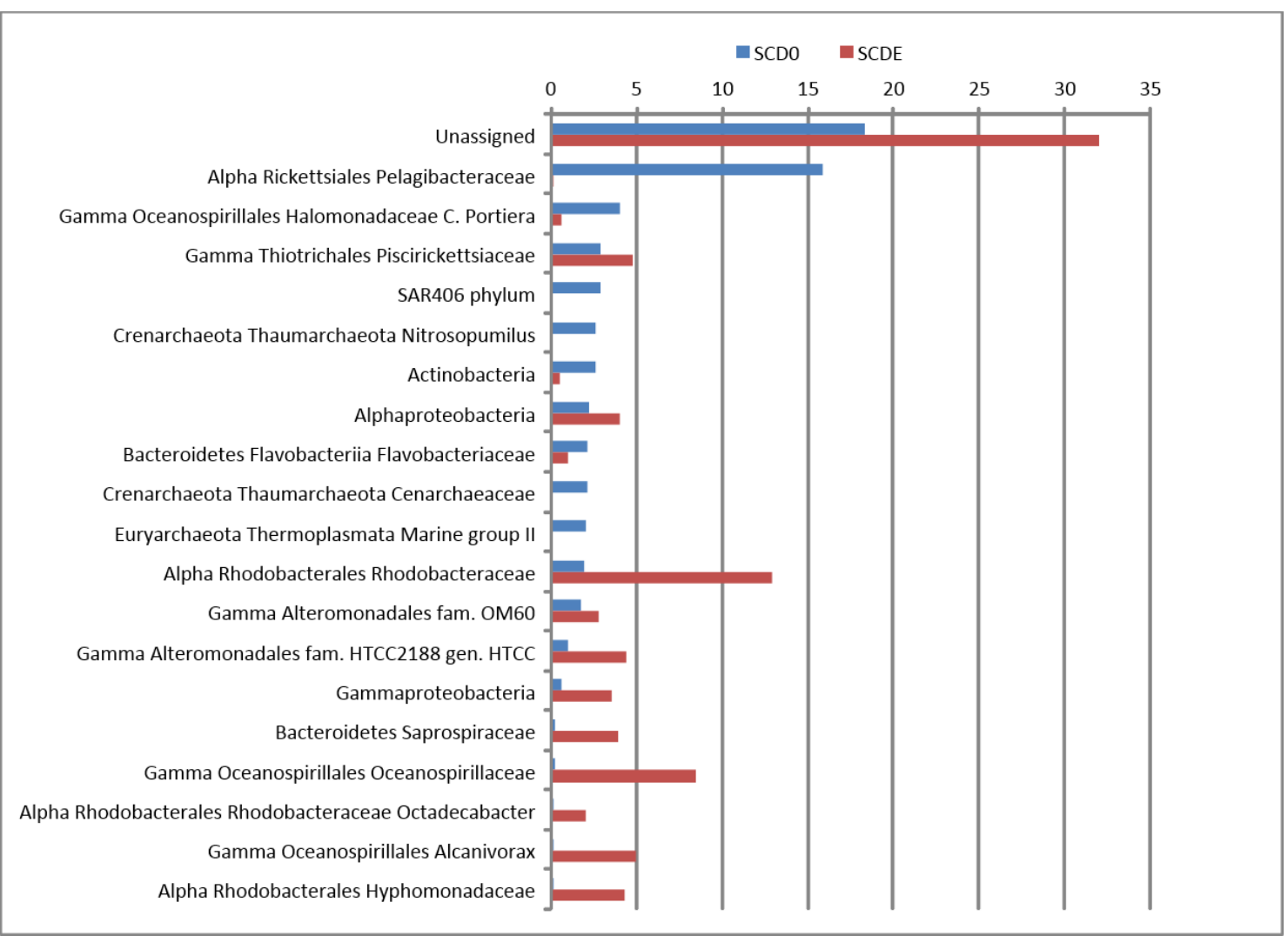

290 Figure 2. Taxonomic composition of the two metagenomes (in percentage). Only taxa with abundance $\geq 2 \%$ in either sample are shown. Taxonomic classification as provided by the phylogenetic analysis of the EBI Metagenomics pipeline (which explains some apparent taxonomic inconsistency such as "Alpha Rickettsiales Pelagibacteraceae", referring to sequences that could be classified down to the family level, and further down "Alphaproteobacteria", referring to sequences that could be classified just at the class level). Alpha, and Gamma are abbreviations for the corresponding classes within the Proteobacteria. 
Percentage metagenomic composition shifts

$-100-80-60-40-20 \quad 0 \quad 20 \quad 40 \quad 6080100$

Crenarchaeota Nitrosopumilus

Crenarchaeota Cenarchaeaceae

Euryarchaeota Marinegroup II

Actinobacteria Acidimicrobiales

Bacteroidetes Flavobacteriaceae

Bacteroidetes Cryomorphaceae

Bacteroidetes Saprospiraceae

Chloroflexi SAR202

Chloroplast Stramenopiles

Chloroplast Mamiellaceae

Alpha Pelagibacteraceae

Alpha Rickettsiales

Alpha Rhodospirillaceae

Alpha Hyphomonadaceae

Alpha Rhodobacteraceae

Alpha Octadecabacter

Alpha Hyphomicrobiaceae

Beta Limnohabitans

Beta Variovorax paradoxus

Beta Comamonadaceae

Beta Methylotenera mobilis

Gamma Alteromonadales...

Gamma Alteromonadales HTCC2188

Gamma Alteromonadales...

Gamma Alteromonadaceae

Gamma Alteromonadales...

Gamma Alteromonadales J115

Gamma Oceanospirillales Alcanivorax

Gamma Oceanospirillales SUP05

Gamma Oceanospirillales...

Gamma Oceanospirillales Halomonas

Gamma Oceanospirillales...

Gamma Oceanospirillales...

Gamma Oceanospirillales...

Gamma Thiotrichales Methylophaga

Gamma Thiohalorhabdales...

Gammaproteobacteria

Gamma Enterobacteriaceae

Delta Sva0853 SAR324

Delta Spirobacillales

Delta Nitrospina

Delta Desulfobulbaceae

Epsilon Arcobacter

SAR406 AB16 ZA3312

SAR406 AB16 SGSH944

SAR406 AB16 SargSea-WGS

SAR406 AB16 ZA3648C AEGEAN_185

SBR1093 A712011

Verrucomicrobia Arctic97B-4

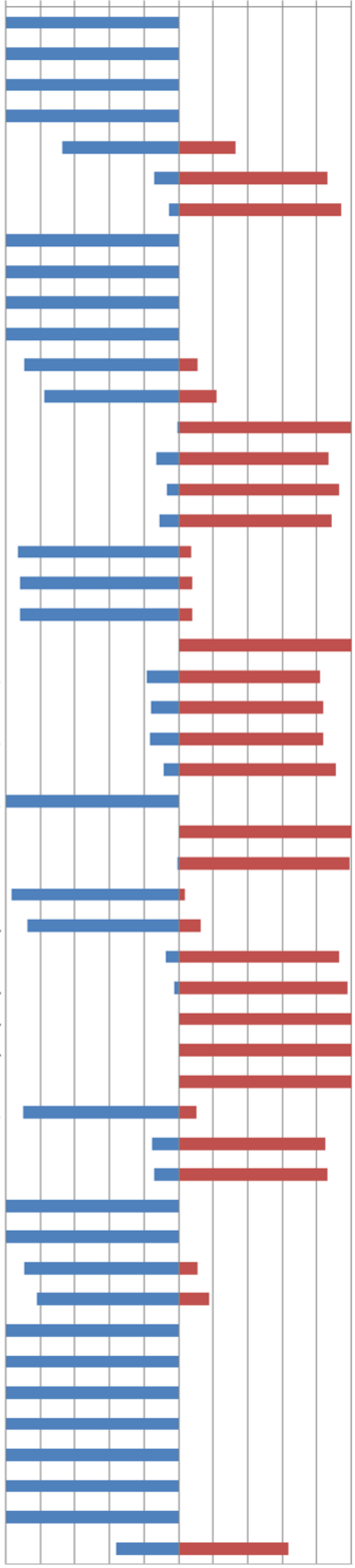

aCD0

- SCDE

Unassigned 
298 Figure 3. Significant shifts in phylogenetic composition observed due to the enrichment. Shown 299 are percentages of the abundance of each taxon in each sample (SCD0 or SCDE) over the total 300 taxon abundance (SCD0 + SCDE). Only statistically significant differences are shown. Taxa are 301 as provided by the phylogenetic analysis of the EBI Metagenomics pipeline (which explains 302 some apparent taxonomic inconsistency, see note in Fig. 2). Alpha, Beta, Gamma, Delta and 303 Epsilon are abbreviations for the corresponding classes within the Proteobacteria.

304 Metagenomic data binning by MetaBat failed to generate discrete bins with sample SCD0: this is 305 not surprising with a diverse natural community under no definite selective pressure where no 306 single species dominate. However, 3 bins were obtained with sample SCDE which by and large 307 matched the results obtained by direct metagenomics analysis. Bin 1, assigned to an Alcanivorax 308 sp. strain, contains genes coding for diagnostic enzymes for the assimilation of $\mathrm{C}_{1}$ carbon 309 through the serine cycle (serine hydroxymethyltransferase, serine-glyoxylate aminotransferase and isocitrate lyase). Bin 2, tentatively classified as the genome of a Hyphomonas sp. strain, also contains genes for the serine cycle (serine hydroxymethyltransferase) and for the anaplerotic ethylmalonyl-CoA and glyoxylate pathways (propionyl-CoA carboxylase, ethylmalonyl-CoA mutase and isocitrate lyase). Bin 3 showed phylogenetic markers mostly associated with genus Methylophaga: it contains genes diagnostic for the ribulose monophosphate (RuMP) cycle (3hexulose-6-phosphate synthase and 6-phospho-3-hexuloisomerase), an alternative metabolic route for the assimilation of $\mathrm{C}_{1}$ carbon, which is the typical metabolic route found in Methylophaga species.

Alpha and beta diversity estimates were based on the phylogenetic data obtained by the EBI Metagenomics analysis. The values are listed in Tables 1 and 2. The effects of the enrichment were an increase in the number of taxa observed, internal diversity $\left(\mathrm{H}^{\prime}\right)$, and evenness. This was due to the sharp decline or disappearance of some of the major taxa composing the original metagenome (all Archaeal groups, algal chloroplast sequences, the Pelagibacteraceae, and the 
323 SAR406 lineages) accompanied by the appearance of a large number of less representative taxa,

324 which shows that the enrichment process was halted at a very early stage, as intended.

325

326 Table 1. Alpha diversity results for samples SCD0 and SCDE based on phylogenetic data.

\begin{tabular}{|l|c|c|}
\hline Alpha diversity indicators & SCD0 & SCDE \\
\hline Shannon index (H') & 3.95 & 4.97 \\
\hline evenness (E) & 0.68 & 0.84 \\
\hline Number of observed taxa & 329 & 363 \\
\hline Chao taxa number estimator & 460.8 & 544.4 \\
\hline
\end{tabular}

328 Table 2. Beta diversity between samples SCD0 and SCDE based on phylogenetic data.

\begin{tabular}{|l|l|}
\hline \multicolumn{2}{|c|}{ Beta diversity indexes } \\
\hline & \\
\hline Bray-Curtis & 0.56 \\
\hline Jaccard & 0.76 \\
\hline Kulczynski & 0.60 \\
\hline Chao index & 0.77 \\
\hline
\end{tabular}

329

330

331

332

333

334

335

336

337

\section{Overall analysis of the functional annotation}

Significant shifts between the functional annotation of samples SCD0 and SCDE obtained by EBI Metagenomics were observed.

The analysis revealed somewhat surprising large increases in categories GO:0000103 "sulfate assimilation" and GO:0008272 "sulfate transport". Among the other categories, significantly rising in hit numbers were GO:0016846 “carbon-sulfur lyase activity”, GO:0016705 "oxidoreductase activity, acting on paired donors, with incorporation or reduction of molecular oxygen” and GO:0004497 “monooxygenase activity”, GO:0051537 "2 iron, 2 sulfur cluster 
338 binding”, GO:0006730 “one-carbon metabolic process”, GO:0004488

339 "methylenetetrahydrofolate dehydrogenase (NADP ${ }^{+}$)" and GO:0008864 "formyltetrahydrofolate

340 deformylase", all of which can be associated to the catabolism of MSA. The latter two may

341 suggest that a selection for organisms that metabolize formaldehyde through the condensation

342 with tetrahydrofolate (rather than with tetrahydromethanopterin) may have occurred. Oddly,

343 though, GO:0046653 "tetrahydrofolate metabolic process" and GO:0046654 "tetrahydrofolate

344 biosynthetic process" were found among the significantly decreased categories. Fittingly, several

345 category associated with oxygenic photosynthesis and GO:0004329 "formate-tetrahydrofolate

346 ligase activity" (incorporation of formate into biomass in anaerobic conditions) also decreased in

347 abundance.

348 Among the monooxygenase genes significantly increased in number during the enrichment, 5

349 hits for gene $m s u D / s s u D$ were found in the assembled metagenomic data of the enriched sample

350 (SCDE), while no homolog was present in sample SCD0.

351 Since no hits for $m s m$ genes were observed in the metagenomic results by either EBI

352 Metagenomics or IMG/MER, the sequence data were also screened locally by hmmsearch

353 (HMMER3 ) and tblastn (McGinnis \& Madden, 2004). The only hits obtained with protein

354 MsmA (expectation value cutoff $10^{-5}$ ) contained short Rieske-associated motifs (spacers varying

355 from 16 to 18 in SCD0, and from 16 to 23 in SCDE) and were low scoring. As for protein

356 MsmE, no hits were found at E-value $\leq 10^{-5}$.

357 In order to complement the metagenomic data, $m s m A$ and $m s m E$ genes were investigated by an

358 amplicon survey experiment. Direct amplification was weak or absent, so a nested PCR approach

359 was adopted for both genes, which witnesses to the overall low concentration of these sequences 
360 in the metagenomes. Amplification of gene A was achieved from both samples. On the contrary,

361 despite repeated and insistent efforts, we were unable to amplify gene E from the enrichment

362 sample (SCDE). For convenience, the three amplicon sets will be designated as follows: SCD0-

363 A ( $m s m A$ amplicons from sample SCD0), SCDE-A ( $m s m A$ amplicons from sample SCDE) and

364 SCD0-E ( $m s m E$ amplicons from sample SCD0). The three amplicon pools were sequenced by

365 ion Torrent technology. The $\mathrm{G}+\mathrm{C}$ content of the processed reads was close to $40 \%$ in all 3 cases

366 (Table S5). Namely, while community metagenome grew from $43.30 \%$ to $53.29 \% \mathrm{G}+\mathrm{C}$ content,

367 there was almost no difference in base composition between of the $m s m A$ reads before and after

368 the enrichment. The reads were then translated and checked for frameshift-generating sequencing

369 errors and all sequences containing an undetermined codon or a stop codon were eliminated.

370 Statistics relative to each case are shown in Table S5.

371 Rarefaction curves constructed from clustering data at a 0.03 distance are represented in Fig. 4.

372 Although a definite plateau is not achieved, gene A looks sampled at satisfactory depth in both

373 SCD0-A and SCDE-A sets. On the contrary, the level of amplification and sequencing of gene E

374 in set SCD0-E was clearly insufficient. These results suggest that a deeper sequencing would be

375 needed. However, it is also true that a huge part of the data was deleted at the quality control step

376 (Table S5) so better sequencing quality rather than deeper sequencing might be the solution. 


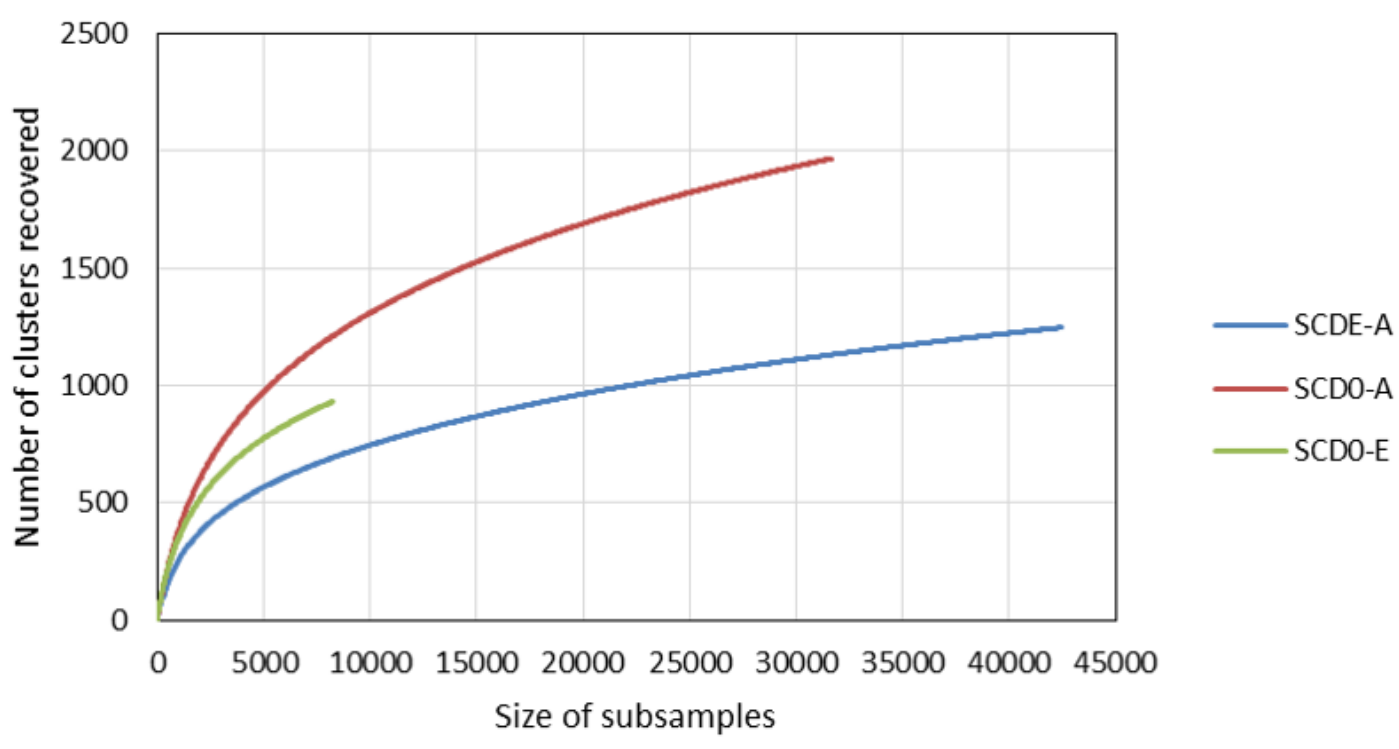

378

Figure 4. Comparison of the rarefaction curves constructed with sequencing data from the amplicon survey experiment. SCD0-A and SCDE-A refer to gene $m s m A$ before and after the enrichment with MSA, respectively. SCD0-E refers to gene $m s m E$ before the enrichment.

The data from clustering of MsmA and MsmE sequences at 0.03 cutoff were analyzed for alpha and beta diversity. Clearly there was an evolution in MsmA sequences during the enrichment process which lead to the loss of some sequence diversity (Table 3). The shift in MsmA allele composition is very significant as illustrated by the high values of the beta diversity indexes $($ Bray Curtis $=0.95 ;$ Jaccard $=0.98 ;$ Kulczynski $=0.95 ;$ Chao index $=0.76)$.

Table 3. Alpha diversity results for $m s m A$ and $m s m E$ genes in samples SCD0 and SCDE.

\begin{tabular}{|c|c|c|c|}
\hline & \multicolumn{2}{|c|}{$\boldsymbol{m s m \boldsymbol { A } \text { gene }}$} & $\boldsymbol{m s m E}$ gene \\
\hline Indexes & SCD0 & SCDE & SCD0 \\
\hline Shannon & 5.08 & 3.56 & 5.32 \\
\hline evenness & 0.66 & 0.49 & 0.78 \\
\hline $\mathrm{S}_{\text {obs }}$ & 2211 & 1410 & 933 \\
\hline Chao estimator & 3073.2 & 2068.6 & 1331.3 \\
\hline
\end{tabular}


393 Conservation analysis was performed with the predicted MsmA and MsmE sequences (Fig.s 5

394 and 6). The edges of the sequences, corresponding to the PCR primers, should be $100 \%$

395

396

397

398

399

400

401

402

403

404

405

conserved. The low conservation levels seen in these regions are artifacts due to the occurrence

of a few shorter sequences in the datasets and lower quality at the end of reaction. In the case of

MsmA (Fig. 5), the levels of amino acid conservation are generally higher and more constant

along the central region of the fragment. If the primers regions are not taken into account, all the conservation values are $\geq 92.35 \%$, with the exception of a one-amino acid trough (a Serine at position 30) with 58\% conservation. Particularly, all the amino acids in the Rieske associated motif and spacer demonstrate conservation levels higher than 99\%. Regarding MsmE (Fig. 6), it is possible to observe regions with high levels of conservation, which in some cases are greater than $99 \%$. However, the overall conservation is much less constant than for MsmA, with 4 positions below $85 \%$. The lowest conservation value (61\%) was found for a Serine at position 10. 


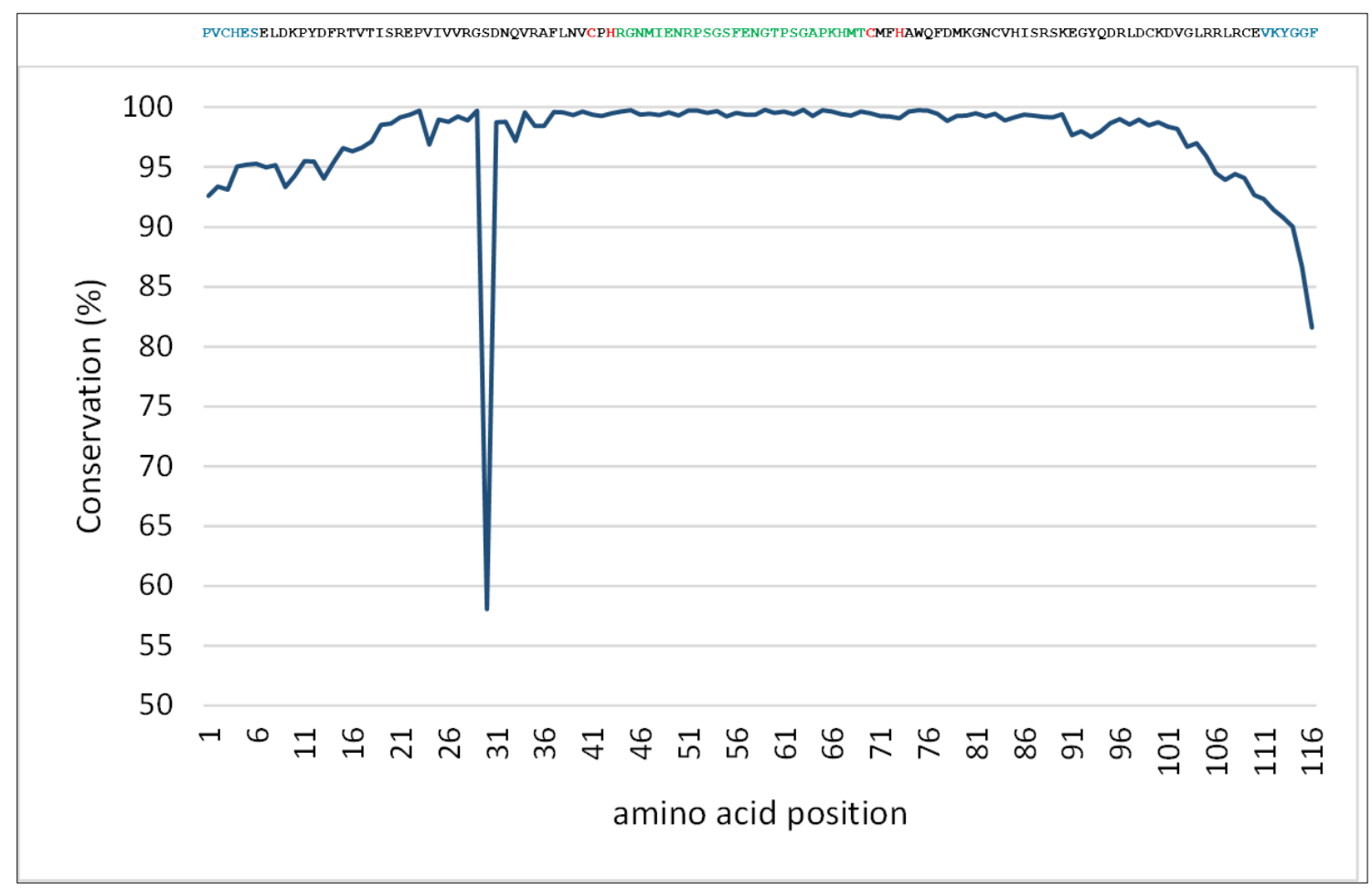

407 Figure 5. Conservation analysis of the predicted MsmA sequences from joined samples SCD0_A 408 and SCDE_A. Displayed on top is the consensus sequence. Amino acids in blue correspond to 409 PCR primers. Amino acids in red correspond to the cysteine and histidine residues typical of the 410 Rieske-associated motif. Amino acids in green represent the characteristic long spacer found in 411 the Rieske motif in MsmA. Low conservation of the beginning and end of the sequence

412 (corresponding to PCR primers) are artifacts explainable by the presence of short reads in the 413 dataset. 


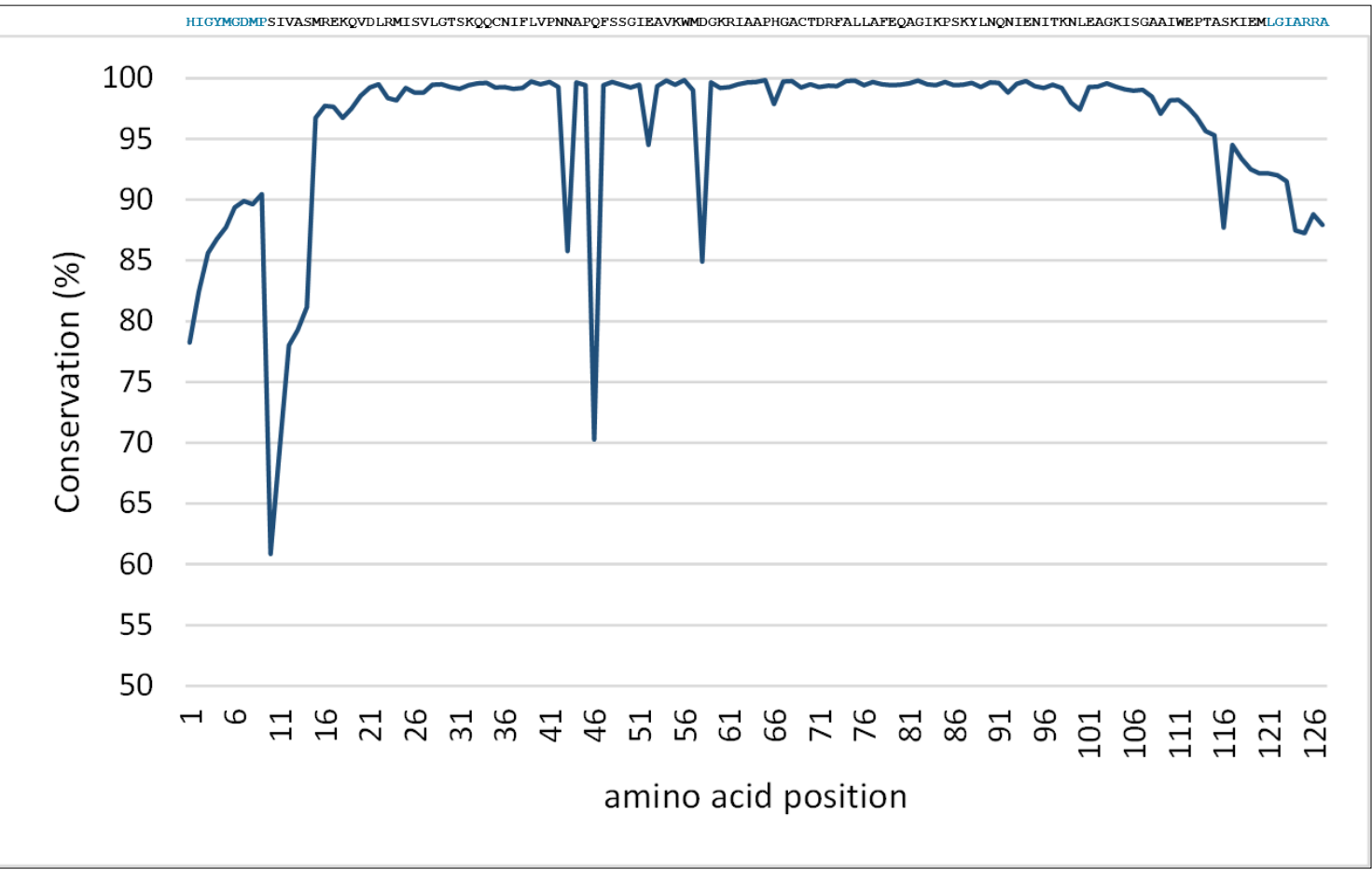

415

416

417

418

419

420

421

422

423

424

425

426

427

428

Figure 6. Conservation analysis of the predicted MsmE sequences from sample SCD0_E. Displayed on top is the consensus sequence. Amino acids in blue correspond to the PCR primers. Low conservation of the beginning and end of the sequence can be explained as in Fig. 5.

\section{Phylogenetic trees of the predicted MsmA and MsmE sequences}

Phylogenetic trees were constructed based on the nucleotide data corresponding to the representative protein sequences of clusters obtained with distance cutoff values 0.15 (for MsmA) or 0.10 (for MsmE). In the process, sequences from this study were aligned with metagenomic seawater homologs obtained in a previous work (Henriques \& De Marco, 2015a), with sequences EF103447, EF103448 and EF103448 from SSM clones (Leitão, MoradasFerreira \& De Marco, 2009), with sequences from the GOS project (Rusch et al., 2007) and with sequences from cultured strains. Phylograms of $m s m A$ and $m s m E$ sequences are shown in Fig.s 7 and 8. Although some degree of caution is required in the analysis of these sequences due to the fact that they were produced by 3 rounds of amplification (whole-genome amplification followed 
429 by nested PCR), the results obtained seem to fit logical expectations. In the case of gene $m s m A$, it 430 is possible to observe a clear separation ( $95 \%$ bootstrap value) between two principal branches:

431 one consists only of metagenomic sequences and includes all the $m s m A$ reads generated in this 432 study; the other group (omitted as root in Fig. 7) contains the sequences from cultured strains 433 (Alpha, Beta or Gammaproteobacteria) of both marine and soil origin. In a similar way, the 434 phylogram for gene $m s m E$ shows an unambiguous split between two major groups (94\% 435 bootstrap value): one comprising mainly metagenomic sequences, including all the $m s m E$ reads 436 from this study, sequences from the GOS project, and two from cultured marine strains $C$.

437 Puniceispirillum marinum IMCC1322 and C. Filomicrobium marinum str. Y; plus a second 438 group containing all other sequences from cultured strains (Alpha and Betaproteobacteria). 


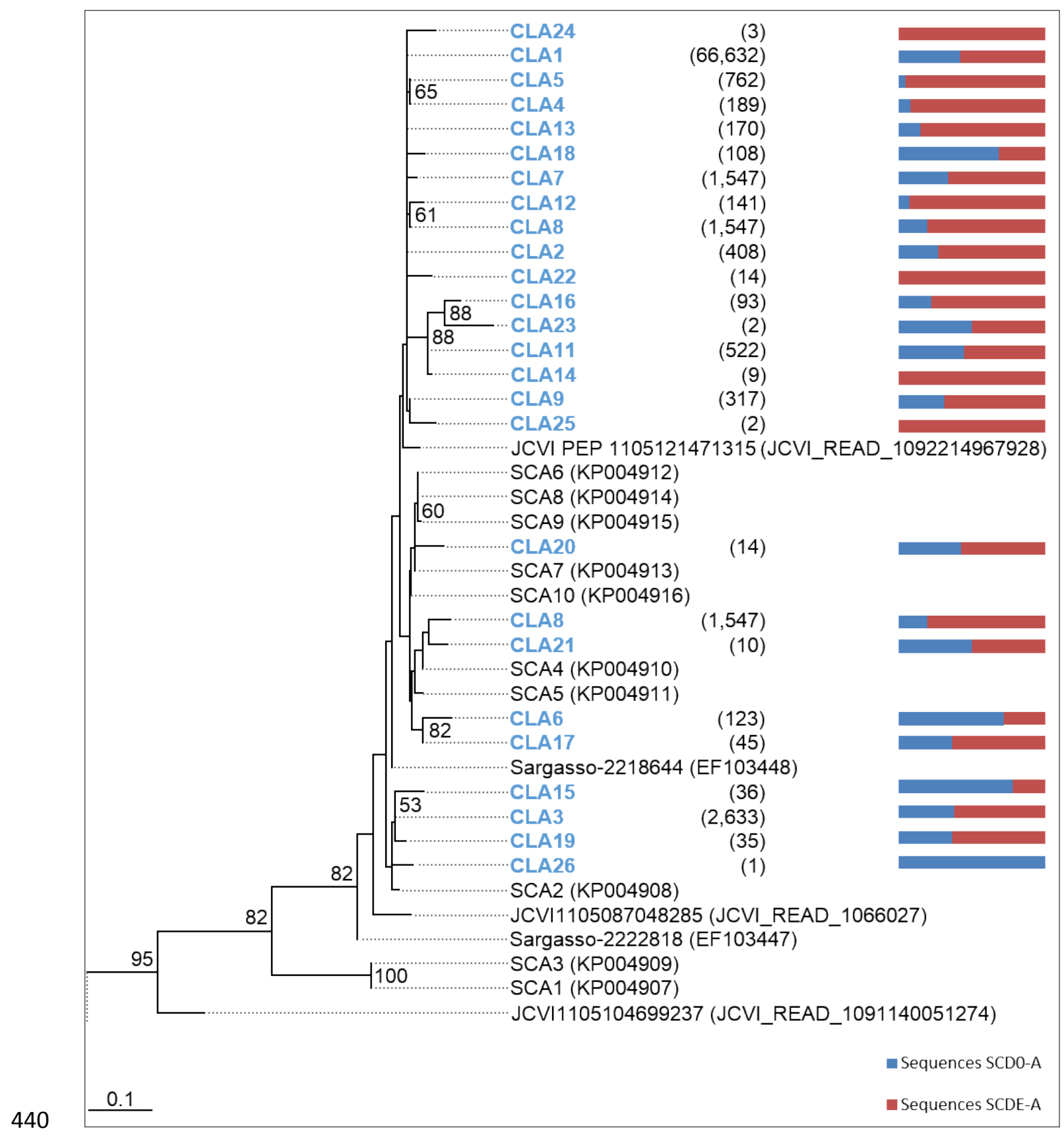

441 Figure 7. Phylogenetic tree of $m s m A$ sequences. Clusters with sequences from this study are in 442 blue: CLA stands for $m s m A$ clusters. Between brackets is the total number of sequences in each 443 cluster. Horizontal bars indicate the relative frequency of sequences of each cluster (SCD0 in 444 blue and SCDE in red). The accession numbers of the sequences previously published are 445 between brackets. The omitted branch is constituted mostly by sequences from genomes of 446 cultured strains. The accession numbers of these sequences are the following: AF354805 447 (Marinosulfonomonas methylotropha str. TR3), KJ789392 (Methylobacterium sp. str. P1), 448 KJ789392 (Hyphomicrobium sp. str. P2), GOS sequence JCVI_READ_2101946, KM879220 (C. 449 Filomicrobium marinum str. Y), NC_011892 (Methylobacterium nodulans ORS 2060), 
450 NC_011894.1 (Methylobacterium nodulans ORS 2060), KJ789395 (Methylobacterium sp. str. 451 RD41), NZ_KB375270 (Afipia felis str. ATCC 53690), EF459501 (Afipia felis str. 25E1), 452 AF091716 (Methylosulfonomonas methylovora str. M2), CP001751 (C. Puniceispirillum 453 marinum str. IMCC1322), NZ_AKCV01000022 (Ralstonia sp. str. PBA), AP014581 454 (Burkholderia sp. str. RPE67), and CP003775 (Burkholderia cepacia str. GG4), CCYE01000041 455 (Pseudomonas xanthomarina str. S11). Nucleotide sequences corresponding to cluster456 representative MsmA sequences obtained at 0.15 distance cutoff were used to infer the 457 phylogenetic tree by Maximum Likelihood with 100 bootstrap iterations. Bootstrap values < $45850 \%$ are omitted.

459

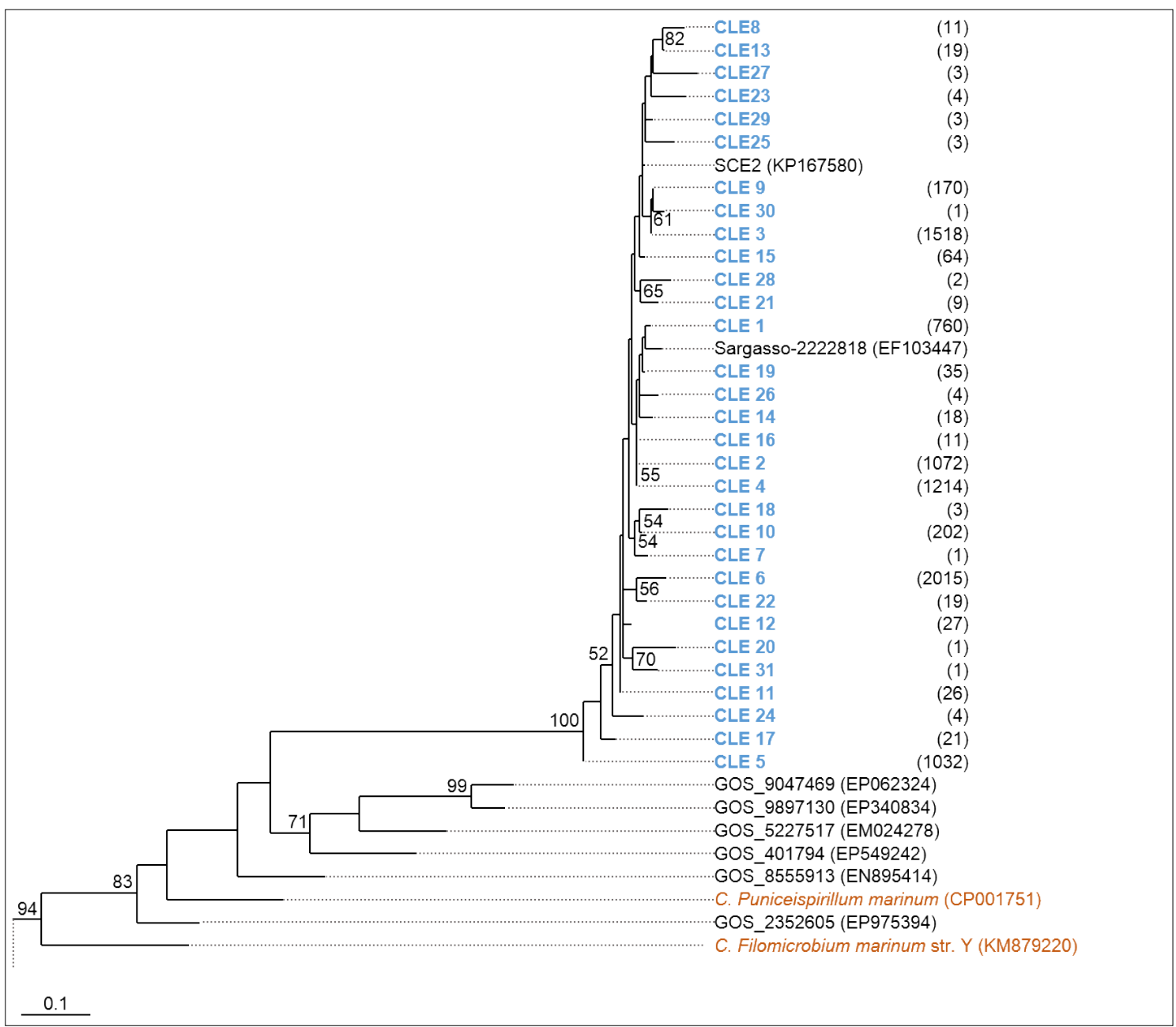

Figure 8. Phylogenetic tree of $m s m E$ sequences. Clusters with sequences from this study are in blue: CLE stands for $m s m E$ clusters. Between brackets is the total number of sequences in each cluster. Sequences from cultured strains are in orange. The accession numbers of the sequences previously published are between brackets. The omitted branch is constituted by sequences from genomes of cultured strains, with the following accession numbers: NZ_AZUP00000000.1 
466 (Methyloversatilis discipulorum str. FAM1), NZ_AFHG01000044 (Methyloversatilis universalis 467 str. FAM5), NZ_ARVV01000001 (Methyloversatilis discipulorum str. RZ18-153), 468 NZ_AKCV01000024 (Ralstonia sp. str. PBA), CCAZ020000001 (Afipia felis genospecies A str. 469 76713), NZ_JNIJ01000008 (Bradyrhizobium sp. str. URHD0069), NZ_KB891326 (Thiobacillus 470 thioparus str. DSM 505), NZ_AQWL01000003 (Thiobacillus denitrificans str. DSM 12475), 471 AZSN01000017 (Methylibium sp. str. T29-B), NC_008825 (Methylibium petroleiphilum str. 472 PM1), NZ_JADL01000017 (Rhodospirillales bacterium str. URHD0088), KP025766

473 (Methylobacterium sp. str. P1), AF091716 (Methylosulfonomonas methylovora str. M2), and 474 KP025767 (Marinosulfonomonas methylotropha str. TR3). Nucleotide sequences corresponding 475 to cluster-representative MsmE sequences obtained at 0.10 distance cutoff were used to infer the 476 phylogenetic tree by Maximum Likelihood with 100 bootstrap iterations. Bootstrap values < $47750 \%$ are omitted. 


\section{Discussion}

479 Genes associated to the degradation of methanesulfonic acid have previously been studied in 480 various bacterial isolates and found in several metagenomic studies. A clear $\mathrm{G}+\mathrm{C}$-content 481 discrepancy between the former and the latter sequences shows that MSA-degrading strains 482 isolated in the laboratory are not good representatives of the natural communities, especially for 483 marine water. For this reason, in this study we tried to obtain a more faithful snapshot of the 484 MSA-affected microbiota by keeping enrichment parameters as close as possible to field 485 conditions and observing the community at an early stage of enrichment. We started from a 486 surface seawater biomass sample and compared metagenomic information from before and after 48716 days of amendment with MSA as the sole organic nutrient. Despite the fact that there was no 488 subculturing, and that the time of enrichment was not sufficient to isolate culturable 489 methylotrophs to purity, the evolution of the community was notorious in the $\mathrm{G}+\mathrm{C}$ content of 490 the metagenomic data, from $43 \%$ of sample SCD0 to $53 \%$ of sample SCDE.

491 Phylogenetically, the enrichment with MSA shifted the prokaryotic community of our sample 492 from a composition fairly typical of oceanic surface waters (mainly Alphaproteobacteria 493 (particularly Pelagibacteraceae), Gammaproteobacteria, Bacteroidetes, SAR406 phylum and 494 Archaea) to a significantly different community with large increases in methylotroph-harboring taxa such as the Rhodobacteraceae, the Piscirickettsiaceae and suspected methylotrophs such as

496 Alcanivorax, accompanied by other emerging taxa not known to harbor methylotrophs 497 (Alteromonadales, Oceanospirillaceae, Hyphomonadaceae). Such a considerable change in 498 phylogenetic profile can be the result of adaptation to using MSA as nutrient, but may also be the 499 sign of a move from an oligotrophic to a more copiotrophic community. 
500 The indications obtained from the analysis of the metagenomic sequences at the functional level

501 showed enrichment in some functions associated with methylotrophic metabolism and in [2Fe-

502 2S] cluster-containing monooxygenases, a category including MSAMO.

503 Binning of the metagenomic sequence data revealed the presence of 3 coherent sets of sequences

504 in sample SCDE matching genera whose abundance was seen increasing in the 16S-based

505 phylogenetic analysis: Alcanivorax, a possible methylotroph, Hyphomonas and Methylophaga, a

506 genus of genuine methylotrophic strains. Genus Hyphomonas is not known to be able of

507 methylotrophic growth. However, genes possibly involved in $\mathrm{C}_{1}$ metabolism were found in the 3

508 bins.

509 Interesting data were also obtained on the two genes associated to the catabolism of MSA, $m s m A$

510 and $m s m E$, by a targeted single-gene high-throughput sequencing strategy. Gene $m s m A$ could not

511 be detected directly in the metagenomic sequence data probably due to an unsuitable ratio

512 between sequencing depth and community complexity. However, after PCR amplification and

513 sequencing, a multitude of diverse sequences was obtained from both the original and enriched

514 sample: despite the shifts in phylogenetic composition caused by the enrichment process, at the

515 level of gene $m s m A$ the $\mathrm{G}+\mathrm{C}$ content remained practically constant. All these sequences from

516 both samples were shown to belong in a very solid metagenomic branch, clearly separate from

517 all the homologs encountered in cultivated strains of both soil and marine origin and

518 unmistakably some sequence clusters were seen shrinking (or even disappearing) or expanding

519 due to the enrichment regime. Gene E too was not found in the metagenomic sequence data of

520 either sample, however we could amplify it from the initial seawater sample (SCD0) with a

521 variety of diverse sequences most closely related to other known metagenomic sequences. Also

522 in this case, our $m s m E$ sequences together with other metagenomic occurrences formed a group 
523 clearly distinct from homologs encountered in soil species, although in this instance clustering

524 with the $m s m E$ genes from two marine cultivated strains (C. Puniceispirillum marinum and C.

525 Filomicrobium str. Y) was observed. Rather surprisingly, we were not able to amplify gene E

526 from the enriched sample (SCDE). This fact suggests that two different populations of bacteria

527 may have existed in sample SCD0: one, harboring both genes A and E, which was wiped out

528 during the enrichment, and another carrying just the MSA monooxygenase (gene A) and

529 importing methanesulfonate from the medium using a transporter alternative to MsmE whose

530 gene cannot be amplified by the primers we employed. Scanning through the functional

531 annotation of the two assembled metagenome sequences, we found 31 "ABC-type

532 nitrate/sulfonate/bicarbonate transport system, periplasmic component" hits in SCD0 and 55 in

533 SCDE: this is the description of a family of MsmE paralogs. It was already recognized that gene

534 E showed a lesser level of conservation than gene A and our data corroborate this idea and

535 indicate that regrettably $m s m E$ cannot be used as a reliable functional indicator of MSA utilizers.

536 An alternative enzyme has been found in non-methylotrophs that use MSA as a source of sulfur:

537 SsuD (also MsuD) is an $\mathrm{FMNH}_{2}$-dependent alkanesulfonate monooxygenase which can

538 desulfonate MSA (Eichhorn, Van Der Ploeg \& Leisinger, 1999; Endoh et al., 2003). Intriguingly,

539 we recently described a Rhododoccus strain that grows on MSA possibly employing SsuD

540 (Henriques \& De Marco, 2015c). The fact that we detected $5 \mathrm{msuD} / \mathrm{ssuD}$ homologs in the

541 enriched metagenome (and none in the original sample) suggests that this alternative sulfonate

542 monooxygenase may be another relevant marker gene for MSA utilization in the oceanic

543 environment.

544 This evidence may also point to two possible alternative scenarios: in the first, at least part of the 545 degradation of methanesulfonate is accomplished by non-methylotrophic bacteria capable of 
546 cleaving the MSA molecule and of using the resulting formaldehyde purely as a source of

547 energy: a similar model has been found at work with $\mathrm{C}_{1}$ compounds such as methanol,

548 formaldehyde, methylamine, trimethylamine, trimethylamine $\mathrm{N}$-oxide and the $\mathrm{C}_{1}$ moiety of

549 glycine betaine and dimethylsulfoniopropionate in marine strain C. Pelagibacter ubique

550 HTCC1062 of the SAR11 clade (Sun et al., 2011) which lead the authors to coin the term

551 'methylovore' for this type of metabolism. In that study, MSA was not a tested compound:

552 indeed, genes $m s m A$ or $s s u D$ are not present in the published genome sequences within the

553 Pelagibacteraceae and we see this family disappear from our enriched sample. However, it is not

554 implausible that other non-methylotrophic marine species may use MSA co-metabolically as a

555 source of energy in an analogous fashion. A second scenario can be imagined of a syntrophic

556 association between non-methylotrophic microorganisms expressing MsmA or SsuD and

557 methylotrophs incapable of cleaving MSA. This hypothesis is especially relevant for marine

558 water where many of the microorganisms are found in suspended particles or flocks (Azam, 559 1998).

560 Due to time and practical constraints, this study reports on the community evolution of a single

561 specific sample. As such, the influence of confounding factors on the outcomes cannot be

562 properly discarded and our results must be considered explorative in nature. However, many of

563 the phylogenetic and metabolic data obtained distinctly suggest that MSA was the factor

564 genuinely causing the differences observed. Gene $m s m A$ appears to be a good marker for MSA

565 degraders, although further study into organisms employing alternative MSA-cleaving enzymes

566 is needed in order to assess the relative importance of the different genes. Enrichment-free

567 strategies such as single-cell genome sequencing, which provides joined functional and

568 phylogenetic data, or MSA-induced metatranscriptomic shift analysis or in situ physiological 
569 studies are necessary to obtain a more complete and realistic picture less dependent on lab

570 culturing.

571 


\section{Acknowledgements}

573 We wish to acknowledge the help received from colleagues at CESPU Patrícia Duarte, Nilza

574 Ribeiro, Claudia Ribeiro and Hugo Ribeiro, and Hassan Bousbaa (IINFACTS) for institutional

575 support. We also thank Ana Toribio (ENA), Hubert Denise (EBI Metagenomics), Benli Chai

576 (RDP), Jennifer Jackson (Galaxy), Matthieu Vizuete-Forster (Illumina), Scot Dowd (Molecular

577 Research LP), the NGS team at Stabvida, I-Min Chen (IMG/JGI), Brandon KB Seah (Max

578 Planck Institute for Marine Microbiology, Bremen, Germany), and Inês Cruz (Faculty of

579 Science, U. of Porto, Portugal) for their patient technical advice. Finally, we wish to

580 acknowledge the helpful criticism by the two anonymous reviewers of this work. 


\section{References}

582

583

584

585

586

587

588

589

590

591

592

593

594

595

596

597

598

599

600

601

602

603

604

605

606

607

608

609

610

611

612

613

614

615

616

617

618

Andreae MO. 1986. The ocean as a source of atmospheric sulfur compounds. In: Patrick Buat-Ménard ed. The role of air-sea exchange in geochemical cycling. 331-362. DOI: 10.1007/978-94-009-4738214.

Anesti V., McDonald IR., Ramaswamy M., Wade WG., Kelly DP., Wood AP. 2005. Isolation and molecular detection of methylotrophic bacteria occurring in the human mouth. Environmental Microbiology 7:1227-1238. DOI: 10.1111/j.1462-2920.2005.00805.x.

Azam F. 1998. Microbial Control of Oceanic Carbon Flux: The Plot Thickens. Science 280:694-696. DOI: $10.1126 /$ science.280.5364.694.

Azra Moosvi S., McDonald IR., Pearce D a., Kelly DP., Wood AP. 2005. Molecular detection and isolation from Antarctica of methylotrophic bacteria able to grow with methylated sulfur compounds. Systematic and Applied Microbiology 28:541-554. DOI: 10.1016/j.syapm.2005.03.002.

Baxter NJ., Scanlan J., De Marco P., Wood AP., Murrell JC. 2002. Duplicate copies of genes encoding methanesulfonate monooxygenase in Marinosulfonomonas methylotropha strain TR3 and detection of methanesulfonate utilizers in the environment. Applied and environmental microbiology 68:28996. DOI: 10.1128/AEM.68.1.289.

Boden R., Thomas E., Savani P., Kelly DP., Wood AP. 2008. Novel methylotrophic bacteria isolated from the River Thames (London, UK). Environmental Microbiology 10:3225-3236. DOI: 10.1111/j.1462-2920.2008.01711.x.

Bray JR., Curtis JT. 1957. An Ordination of the upland forest community of southern Wisconsin. Ecology Monographs 27:325-349. DOI: 10.2307/1942268.

Chao A. 1984. Nonparametric Estimation of the Number of Classes in a Population. The Annals of Mathematical Statistics 20:572-579. DOI: 10.1214/aoms/1177729949.

Chao A., Chazdon RL., Shen TJ. 2005. A new statistical approach for assessing similarity of species composition with incidence and abundance data. Ecology Letters 8:148-159. DOI: 10.1111/j.14610248.2004.00707.x.

Charlson RJ., Lovelock JE., Andreae MO., Warren SG. 1987. Oceanic phytoplankton, atmospheric sulphur, cloud albedo and climate. Nature 326:655-661. DOI: 10.1038/326655a0.

Dereeper A., Guignon V., Blanc G., Audic S., Buffet S., Chevenet F., Dufayard JF., Guidon S., Lefort V., Lescot M., Claverie JM., Gascuel O. 2008. Phylogeny.fr: robust phylogenetic analysis for the nonspecialist. Nucleic Acids Research 36:465-469. DOI: 10.1093/nar/gkn180.

Eddy SR. 2011. Accelerated Profile HMM Searches. PLoS Computational Biology 7:e1002195. DOI: 10.1371/journal.pcbi.1002195.

Edgar RC., Haas BJ., Clemente JC., Quince C., Knight R. 2011. UCHIME improves sensitivity and speed of chimera detection. Bioinformatics 27:2194-2200. DOI: 10.1093/bioinformatics/btr381.

Eichhorn E., Van Der Ploeg JR., Leisinger T. 1999. Characterization of a two-component alkanesulfonate monooxygenase from Escherichia coli. Journal of Biological Chemistry 274:26639-26646. DOI: 10.1074/jbc.274.38.26639. 
619

620

621

622

623

624

625

626

627

628

629

630

631

632

633

634

635

636

637

638

639

640

641

642

643

644

645

646

647

648

649

650

651

652

653

654

655

656

657

Endoh T., Kasuga K., Horinouchi M., Yoshida T., Habe H., Nojiri H., Omori T. 2003. Characterization and identification of genes essential for dimethyl sulfide utilization in Pseudomonas putida strain DS1. Applied Microbiology and Biotechnology 62:83-91. DOI: 10.1007/s00253-003-1233-7.

Faith DP., Minchin PR., Belbin L. 1987. Compsitional dissimilarity as a robust measure of ecogical distance. Vegetatio 69:57-68. DOI: 10.1007/BF00038687.

Fish J a., Chai B., Wang Q., Sun Y., Brown CT., Tiedje JM., Cole JR. 2013. FunGene: The functional gene pipeline and repository. Frontiers in Microbiology 4:1-14. DOI: 10.3389/fmicb.2013.00291.

Gifford SM., Sharma S., Booth M., Moran MA. 2013. Expression patterns reveal niche diversification in a marine microbial assemblage. The ISME journal 7:281-98. DOI: 10.1038/ismej.2012.96.

Gondwe M., Krol M., Gieskes W., Klaassen W., de Baar H. 2003. The contribution of ocean-leaving DMS to the global atmospheric burdens of DMS, MSA, SO2, and NSS SO4. Global Biogeochemical Cycles 17. DOI: 10.1029/2002GB001937.

Guindon S., Dufayard JF., Lefort V., Anisimova M., Hordijk W., Gascuel O. 2010. New algorithms and methods to estimate maximum-likelihood phylogenies: Assessing the performance of PhyML 3.0. Systematic Biology 59:307-321. DOI: 10.1093/sysbio/syq010.

Henriques AC., De Marco P. 2015a. Methanesulfonate (MSA) catabolic genes from marine and estuarine bacteria. PLoS ONE. DOI: 10.1371/journal.pone.0125735.

Henriques AC., De Marco P. 2015b. Complete Genome Sequences of Two Strains of "Candidatus Filomicrobium marinum ," a Methanesulfonate-Degrading Species. Genome Announcements 3:1-2. DOI: 10.1128/genomeA.00160-15.Copyright.

Henriques AC., De Marco P. 2015c. Genome Sequence of Rhodococcus sp. Strain RD6.2 DSM 46800, a Methanesulfonate-Degrading Strain. Genome Announcements 3:9-10. DOI: 10.1128/genomeA.00326-15.Copyright.

Huson D., Mitra S., Ruscheweyh H. 2011. Integrative analysis of environmental sequences using MEGAN4. Genome Research 21:1552-1560. DOI: 10.1101/gr.120618.111.Freely.

Hynes AJ., Wine PH., Semmes DH. 1986. kinetics and mechanism of OH reactions with organic sulfides. J. Phys. Chem 90:4148-4156. DOI: 10.1021/j100408a062.

Inag IP. 2009. Manual para a avaliação da qualidade biológica da água em lagos e albufeiras segundo a Directiva Quadro da Água. Protocolo de amostragem e análise para o Fitoplâncton. Ministério do Ambiente, do Ordenamento do Território e do Desenvolvimento Regional. Instituto da Água, I.P.

Jaccard P. 1912. THE DISTRIBUTION OF THE FLORA IN THE ALPINE ZONE. New Phytologist 11:37-50. DOI: 10.1111/j.1469-8137.1912.tb05611.x.

Jamshad M., De Marco P., Pacheco CC., Hanczar T., Murrell JC. 2006. Identification, mutagenesis, and transcriptional analysis of the methanesulfonate transport operon of Methylosulfonomonas methylovora. Applied and environmental microbiology 72:276-83. DOI: 10.1128/AEM.72.1.276283.2006.

Jeffrey SW., Humphrey GF. 1975. New spectrophotometric equations for determining chlorophylls a, b, c1 and c2 in higher plants, algae and natural phytoplankton. Biochemical Physiology Pflanz:191194. DOI: 10.1016/0022-2860(75)85046-0. 
658

659

660

661

662

663

664

665

666

667

668

669

670

671

672

673

674

675

676

677

678

679

680

681

682

683

684

685

686

687

688

689

690

691

692

693

694

695

696

697

Kang DD., Froula J., Egan R., Wang Z. 2015. MetaBAT, an efficient tool for accurately reconstructing single genomes from complex microbial communities. PeerJ 3:e1165. DOI: 10.7717/peerj.1165.

Kelly DP., Baker SC. 1990. The organosulphur cycle : aerobic and anaerobic processes leading to turnover of C1-Sulphur compounds. FEMS Microbiology 87:241-246. DOI: 10.1016/03781097(90)90461-X.

Kelly DP., Murrell JC. 1999. Microbial metabolism of methanesulfonic acid. Arch Microbiol 5:341-348. DOI: $10.1007 / \mathrm{s} 002030050770$.

Koga S., Tanaka H. 1993. numerical study of the oxidation process of dimethylsulfide in the marine atmosphere. Jornal of Atmospheric Chemistry 17:201-228. DOI: 10.1007/BF00694398.

Legrand M., Feniet-Saigne C. 1991. Methanesulfonic acid in soth polar snow layers: a record of strong El Nino? Geophysical Research 18:187-190. DOI: 10.1029/90GL02784.

Leitão E., Moradas-Ferreira P., De Marco P. 2009. Evidence of methanesulfonate utilizers in the Sargasso Sea metagenome. Journal of basic microbiology 49:S24-30. DOI: 10.1002/jobm.200800223.

Li D., Liu C-M., Luo R., Sadakane K., Lam T-W. 2015. MEGAHIT: An ultra-fast single-node solution for large and com- plex metagenomics assembly via succinct de Bruijn graph. Bioinformatics 31. DOI: 10.1093/bioinformatics/btv033.

Loewenstein Y., Portugaly E., Fromer M., Linial M. 2008. Efficient algorithms for accurate hierarchical clustering of huge datasets: tackling the entire protein space. Bioinformatics (Oxford, England) 24:i41-i49. DOI: 10.1093/bioinformatics/btn174.

Madhaiyan M., Poonguzhali S., Lee J-S., Lee KC., Sundaram S. 2010. Flavobacterium glycines sp. nov., a facultative methylotroph isolated from the rhizosphere of soybean. International journal of systematic and evolutionary microbiology 60:2187-92. DOI: 10.1099/ijs.0.014019-0.

De Marco P., Moradas-Ferreira P., Higgins TP., McDonald I., Kenna EM., Murrell JC. 1999. Molecular analysis of a novel methanesulfonic acid monooxygenase from the methylotroph Methylosulfonomonas methylovora. Journal of bacteriology 181:2244-51.

De Marco P., Murrell JC., Bordalo AA., Moradas-Ferreira P. 2000. Isolation and characterization of two new methanesulfonic acid-degrading bacterial isolates from a Portuguese soil sample. Archives of microbiology 173:146-53. DOI: 10.1007/s002039900124.

De Marco P., Pacheco CC., Figueiredo AR., Moradas-Ferreira P. 2004. Novel pollutant-resistant methylotrophic bacteria for use in bioremediation. FEMS microbiology letters 234:75-80. DOI: 10.1016/j.femsle.2004.03.010.

Markowitz VM., Chen I-MA., Chu K., Szeto E., Palaniappan K., Pillay M., Ratner A., Huang J., Pagani I., Tringe S., Huntemann M., Billis K., Varghese N., Tennessen K., Mavromatis K., Pati A., Ivanova NN., Kyrpides NC. 2014. IMG/M 4 version of the integrated metagenome comparative analysis system. Nucleic Acids Research 42:D568-D573. DOI: 10.1093/nar/gkt919.

McGinnis S., Madden TL. 2004. BLAST: At the core of a powerful and diverse set of sequence analysis tools. Nucleic Acids Research 32:20-25. DOI: 10.1093/nar/gkh435.

Meyer F., Paarmann D., D’Souza M., Olson R., Glass E., Kubal M., Paczian T., Rodriguez A., Stevens R., Wilke A., Wilkening J., Edwards R. 2008. The metagenomics RAST server-a public resource for the automatic phylo- genetic and functional analysis of metagenomes. BMC bioinformatics 9 . 
Mihalopoulos N., Nguyen BC., Boissard C., Putaud JP., Belviso S. 1992. Field study of dimethylsulfide oxidation in the boundary layer: variations of dimethylsulfide, methanesulfonic acid, sulfur dioxide, non-sea-salt sulfate and aitken nuclei at a coastal site. Journal of Atmospheric Chemistry 14:459477. DOI: $10.1007 / \mathrm{BF} 00115251$.

Mitchell A., Bucchini F., Cochrane G., Denise H., Hoopen P t., Fraser M., Pesseat S., Potter S., Scheremetjew M., Sterk P., Finn RD. 2015a. EBI metagenomics in 2016 - an expanding and evolving resource for the analysis and archiving of metagenomic data. Nucleic Acids Research 44:D595-603. DOI: 10.1093/nar/gkv1195.

Mitchell A., Chang H-Y., Daugherty L., Fraser M., Hunter S., Lopez R., McAnulla C., McMenamin C., Nuka G., Pesseat S., Sangrador-Vegas A., Scheremetjew M., Rato C., Yong S-Y., Bateman A., Punta M., Attwood TK., Sigrist CJA., Redaschi N., Rivoire C., Xenarios I., Kahn D., Guyot D., Bork P., Letunic I., Gough J., Oates M., Haft D., Huang H., Natale DA., Wu CH., Orengo C., Sillitoe I., Mi H., Thomas PD., Finn RD. 2015b. The InterPro protein families database: the classification resource after 15 years. Nucleic Acids Research 43:D213-D221. DOI: 10.1093/nar/gku1243.

Moosvi SA., Pacheco CC., McDonald IR., De Marco P., Pearce DA., Kelly DP., Wood AP. 2005. Isolation and properties of methanesulfonate-degrading Afipia felis from Antarctica and comparison with other strains of A. felis. Environmental microbiology 7:22-33. DOI: 10.1111/j.14622920.2004.00661.x.

Mulder CPH., Dimitrakopoulos PG., Hector A., Schmid B. 2004. Species evenness and productivity in experimental plant communities. OIKOS 107:50-63. DOI: 10.1111/j.0030-1299.2004.13110.x.

Neufeld JD., Chen Y., Dumont MG., Murrell JC. 2008. Marine methylotrophs revealed by stable-isotope probing, multiple displacement amplification and metagenomics. Environmental Microbiology 10:1526-1535. DOI: 10.1111/j.1462-2920.2008.01568.x.

Oh H-M., Kwon KK., Kang I., Kang SG., Lee J-H., Kim S-J., Cho J-C. 2010. Complete genome sequence of "Candidatus Puniceispirillum marinum" IMCC1322, a representative of the SAR116 clade in the Alphaproteobacteria. Journal of bacteriology 192:3240-1. DOI: 10.1128/JB.00347-10.

Oksanen AJ., Blanchet FG., Kindt R., Minchin PR., O’Hara RB., Simpson GL., Solymos P., Stevens MHH., Wagner H. 2015. vegan: Community Ecology (R package version 2.3-2). Available at http://CRAN.R-project.org/package=vegan.

Overbeek R., Begley T., Butler RM., Choudhuri J V., Chuang HY., Cohoon M., de Cr??cy-Lagard V., Diaz N., Disz T., Edwards R., Fonstein M., Frank ED., Gerdes S., Glass EM., Goesmann A., Hanson A., Iwata-Reuyl D., Jensen R., Jamshidi N., Krause L., Kubal M., Larsen N., Linke B., McHardy AC., Meyer F., Neuweger H., Olsen G., Olson R., Osterman A., Portnoy V., Pusch GD., Rodionov DA., R??1;ckert C., Steiner J., Stevens R., Thiele I., Vassieva O., Ye Y., Zagnitko O., Vonstein V. 2005. The subsystems approach to genome annotation and its use in the project to annotate 1000 genomes. Nucleic Acids Research 33:5691-5702. DOI: 10.1093/nar/gki866.

Rusch DB., Halpern AL., Sutton G., Heidelberg KB., Williamson S., Yooseph S., Wu D., Eisen J a., Hoffman JM., Remington K., Beeson K., Tran B., Smith H., Baden-Tillson H., Stewart C., Thorpe J., Freeman J., Andrews-Pfannkoch C., Venter JE., Li K., Kravitz S., Heidelberg JF., Utterback T., Rogers Y-H., Falcón LI., Souza V., Bonilla-Rosso G., Eguiarte LE., Karl DM., Sathyendranath S., Platt T., Bermingham E., Gallardo V., Tamayo-Castillo G., Ferrari MR., Strausberg RL., Nealson 
741

742

743

744

745

746

747

748

749

750

751

752

753

754

755

756

757

758

759

760

761

762

763

764

765

766

767

768

769

770

771

772

773

774

775

776

777

778

779

780

K., Friedman R., Frazier M., Venter JC. 2007. The Sorcerer II Global Ocean Sampling expedition: northwest Atlantic through eastern tropical Pacific. PLoS biology 5:e77. DOI: 10.1371/journal.pbio.0050077.

Schäfer H. 2007. Isolation of Methylophaga spp. from marine dimethylsulfide-degrading enrichment cultures and identification of polypeptides induced during growth on dimethylsulfide. Applied and Environmental Microbiology 73:2580-2591. DOI: 10.1128/AEM.02074-06.

Shannon CE. 1948. A Mathematical Theory of Communication. Bell System Technical Journal 27:379423. DOI: 10.1002/j.1538-7305.1948.tb01338.x.

Sun J., Steindler L., Thrash JC., Halsey KH., Smith DP., Carter AE., Landry ZC., Giovannoni SJ. 2011. One Carbon Metabolism in SAR11 Pelagic Marine Bacteria. PLoS ONE 6:e23973. DOI: 10.1371/journal.pone.0023973.

Sunagawa S., Coelho LP., Chaffron S., Kultima JR., Labadie K., Salazar G., Djahanschiri B., Zeller G., Mende DR., Alberti A., Cornejo-castillo FM., Costea PI., Cruaud C., Ovidio F., Engelen S., Ferrera I., Gasol JM., Guidi L., Hildebrand F., Kokoszka F., Lepoivre C. 2015. Structure and function of the global ocean microbiome. Science 348:1-10. DOI: 10.1126/science.1261359.

Swan BK., Tupper B., Sczyrba A., Lauro FM., Martinez-Garcia M., Gonzalez JM., Luo H., Wright JJ., Landry ZC., Hanson NW., Thompson BP., Poulton NJ., Schwientek P., Acinas SG., Giovannoni SJ., Moran MA., Hallam SJ., Cavicchioli R., Woyke T., Stepanauskas R. 2013. Prevalent genome streamlining and latitudinal divergence of planktonic bacteria in the surface ocean. Proceedings of the National Academy of Sciences 110:11463-11468. DOI: 10.1073/pnas.1304246110.

Thompson AS., Owens NJP., Murrell JC. 1995. Isolation and characterization of methanesulfonic Aciddegrading bacteria from the marine environment. Applied and environmental microbiology 61:2388-2393.

Todd JD., Curson ARJ., Kirkwood M., Sullivan MJ., Green RT., Johnston AWB. 2011. DddQ, a novel, cupin-containing, dimethylsulfoniopropionate lyase in marine roseobacters and in uncultured marine bacteria. Environmental Microbiology 13:427-438. DOI: 10.1111/j.1462-2920.2010.02348.x.

Wang Q., Quensen JFI., Fish JA., Lee TK., Sun Y., Tiedje JM., Cole JR. 2013. Ecological Patterns of nifH Genes in Four Terrestrial Climatic Zones. mBio 4:1-9. DOI: 10.1128/mBio.00592-13.Editor.

Waterhouse AM., Procter JB., Martin DMA., Clamp M., Barton GJ. 2009. Jalview Version 2--a multiple sequence alignment editor and analysis workbench. Bioinformatics 25:1189-1191. DOI: 10.1093/bioinformatics/btp033.

White JR., Nagarajan N., Pop M. 2009. Statistical methods for detecting differentially abundant features in clinical metagenomic samples. PLoS computational biology 5:e1000352. DOI: 10.1371/journal.pcbi.1000352.

Whung PY., Saltzman ES., Spencer MJ., Mayewski PA. 1994. Two-hundred-year record of biogenic sulfur in a south greenland ice core (20D). Journal of Geophysical Research 99:1147-1156. DOI: DOI: 10.1029/93JD02732.

Wood DE., Salzberg SL. 2014. Kraken: ultrafast metagenomic sequence classification using exact alignments. Genome biology 15:R46. DOI: 10.1186/gb-2014-15-3-r46.

Zaharia M., Bolosky WJ., Curtis K., Fox A., Patterson D., Shenker S., Stoica I., Karp RM., Sittler T. 
781 2011. Faster and More Accurate Sequence Alignment with SNAP.

782 